\title{
Configural Processing in the Perception of Apparent Biological Motion
}

\author{
Sheba Heptulla Chatterjee \\ Rutgers-The State University of New Jersey \\ Jennifer J. Freyd \\ University of Oregon \\ Maggie Shiffrar \\ Rutgers-The State University of New Jersey
}

\begin{abstract}
In classic demonstrations of apparent motion, observers typically report seeing motion along the shortest possible path between 2 sequentially presented objects. However, when realistic photographs of a human body are sequentially presented at slow temporal rates, observers report paths of apparent motion that are consistent with the movement limitations of the human body even when those paths are not the shortest possible. The current set of experiments examined those aspects of the human form that lead to the perception of biomechanically consistent paths of motion. The authors' findings suggest that the perception of apparent biological motion extends to human movements that involve inanimate objects. The authors also report that observers can perceive apparent motion of nonbiological objects in a manner similar to apparent motion of human bodies. However, a global hierarchy of orientation and position cues resembling the human form is required for the perception of these paths.
\end{abstract}

Our successful interaction with the environment depends largely on our ability to interpret visual forms and motions accurately and rapidly. This ability appears to be particularly important for the visual perception of human movement. Johansson and his colleagues generated extensive interest in the perception of human movement with the demonstration that even under extremely reduced conditions, observers could readily identify human locomotion (Johansson, 1973; 1975; Johansson, von Hofsten, \& Jansson, 1980). More specifically, Johansson (1973) filmed humans moving in the dark with small light sources attached to their joints. When people viewed these films, consisting solely of approximately a dozen moving points of light, they readily recognized the actions performed by the point-light defined actors. Johansson (1973) coined the term "biological motion" to refer to the locomotion of terrestrial animals. Our impressive sensitivity to biological motion is further evidenced by the finding that observers can recognize friends, differentiate gender, categorize locomotive, social, and instrumental actions, as well as identify certain

Sheba Heptulla Chatterjee and Maggie Shiffrar, Department of Psychology, Rutgers--The State University of New Jersey; Jennifer J. Freyd, Department of Psychology, University of Oregon.

This research was funded by National Eye Institute Grant 099310. The results from Experiments 1 and 2 were first presented at the 1993 Psychonomics Conference, Washington, D.C. We thank Laura Lichtey for assistance in creating the stimuli and Alan Gilchrist for providing the wood planks and wood cutting equipment. We also thank David Gilden and Randy Blake for numerous helpful comments on a draft of this article.

Correspondence concerning this article should be addressed to Sheba Heptulla Chatterjee, Department of Psychology, RutgersThe State University of New Jersey, 101 Warren Street, Newark, New Jersey 07102 . Electronic mail may be sent via Internet to sheeba@pegasus.rutgers.edu. dispositional characteristics of a person from point-light information (Dittrich 1993; Kozlowski \& Cutting, 1977; 1978; MacArthur \& Baron, 1983). This sensitivity to biological motion may also extend to human movements or interactions with inanimate objects. For example, observers can estimate the weights of lifted objects when point lights are attached to the objects and the human form lifting them (Runeson \& Frykholm 1981). Moreover, developmental studies indicate that sensitivity to biological motion develops as early as 3 months of age (Bertenthal \& Pinto, 1993).

Orientation and position cues appear to be critically important in the perception of biological forms from pointlight displays. For example, when point lights are mounted off the joints, recognition of the point-light walkers becomes slightly less accurate (Cutting, 1981; Dittrich, 1993). Furthermore, when a point-light walker film is displayed upside down, observers are much less likely to recognize the walker (Barclay, Cutting, \& Kozlowski, 1978; Sumi, 1984). Similarly, adult observers can detect an upright but not an upside down point-light walker presented in a mask of scrambled moving dots (Cutting, Moore, \& Morrison, 1988; Bertenthal \& Pinto, 1994). Sensitivity to biological motion is not limited to humans recognizing other point-light humans. Human observers can also identify the locomotion of many different animals, including baboons, camels, elephants, and horses from dynamic point-light displays (Mather \& West, 1993). Finally, nonprimate animals appear to be sensitive to upright, point-light displays of animal locomotion (Blake, 1993).

Johansson (1973) reported that although observers could readily recognize dynamic point-light displays, they were rarely able to recognize the human form in a static display. This finding suggests that in highly reduced point-light walker displays, motion is required for the extraction of 
human form. Following this, most subsequent research has examined the perception of biological motion using a structure-from-motion approach. Johansson (1973) proposed that the visual system conducts a perceptual vector analysis that decomposes global object or human motion into its common and relative velocities. Other theories have emphasized the importance of global spatial relations or geometric information (Cutting, 1987). For example, Cutting and Proffitt (1981) proposed that the analysis of biological motion proceeds in a hierarchical fashion in which more central limbs are used as a frame of reference to interpret the motion of more distal limbs. Yet other approaches have emphasized the use of constraints, or prior assumptions regarding the nature of the physical world. Bertenthal and Pinto (1994) suggested that human observers use the relative phase between-limb movements to constrain the perception of point-light displays. Several computational approaches have used pairwise rigidity as a necessary constraint to correctly recover the pattern of point-light connectivity and direct the identification of biological movements (Hoffman \& Flinchbaugh, 1982; Webb \& Aggarwal, 1982). Occlusion also appears to constrain the perception of biological motion for both adult and infant observers by facilitating the accurate interpretation of pointlight displays (Bertenthal, Proffitt, Spetner, \& Thomas, 1985; Proffitt, Bertenthal, \& Roberts, 1984).

Whereas the above studies are based on a structure-frommotion perspective, recent research suggests that a motionfrom-structure approach can also yield important insights into our perception of biological motion (Shiffrar, 1994). In other words, biological forms may also influence the perception of biological movements.

Traditionally, the perceptual influence of form on motion has been studied with an apparent motion paradigm. In classic demonstrations of apparent motion, two spatially separated objects are sequentially presented within a certain temporal range so that they give rise to the perception of a single-moving object (Korte, 1915; Wertheimer, 1912). Although an infinite number of possible motion paths connects these two objects, observers typically report seeing only the shortest possible path. Numerous apparent motion studies have suggested that perceived paths of apparent motion are not influenced by the stimulus form. For example, Burt and Sperling (1981) varied the stimulus dimensions of shape, size, and orientation and found no effect on the perceived paths of apparent motion. Moreover, Navon (1976) found that apparent motion was no more likely to be seen between similarly shaped objects as between differently shaped objects.

Other research has suggested that some form manipulations can influence motion percepts. In one experiment, Kolers and Pomerantz (1971) presented observers with two views of the same object in two different orientations. The shortest possible path of motion connecting these images required the perception of a nonrigidly deforming object. However, there also existed a longer path that conserved object form. These researchers found that at sufficiently long temporal durations, observers perceived the relatively long path of apparent motion that was consistent with the rigid rotation of the object. Other studies have also indicated that during sufficiently slow display rates, percepts of apparent motion are consistent with global or higher level constraints. At long temporal durations or stimulus onset asynchronies (SOAs), percepts of apparent motion are influenced by occlusion (Anstis \& Ramachandran, 1985), figural rigidity (Gerbino, 1984), global organization (Pantle \& Petersik, 1980), size and slant (Mack, Klein, Hill, \& Palumbo, 1989), orientation (Foster, 1975; Hecht \& Proffitt, 1991; McBeath \& Shepard, 1989; Proffitt, Gilden, Kaiser, \& Whelan, 1988), surface characteristics (He \& Nakayama, 1994), spatial frequency (Green, 1986), and kinematic geometry (Shepard, 1984).

The use of biological forms also appears to constrain perceived paths of apparent motion. At sufficiently slow display rates, observers tend to see those paths of motion that are consistent with the normal movements of the human body (Shiffrar \& Freyd, 1990). These longer paths always preserve the movement limitations of the human body in terms of its solidity (one part of the body cannot pass through another) and joints (human joints allow a restricted range of movements). Subsequent studies indicated that the perception of these longer, biomechanically consistent paths of motion were not simply a function of increasing SOAs. Rather, observers became increasingly likely to perceive the biomechanically consistent paths, rather than simply longer paths, with increasing SOAs (Shiffrar \& Freyd, 1993).

The purpose of the present series of studies is to identify which aspects of biological forms give rise to the perception of biological motion. We approach this question by examining the generality of this sensitivity and the extent to which it can be explained by the involvement of lower level constraints such as limb orientation, solidity, and surface curvature. To investigate the importance of different properties of biological forms on the perception of biological motion, we used an apparent motion paradigm. Observers viewed sequentially presented photographs of the human body or an inanimate figure in different poses. A competitive strategy was used to evaluate the influence of different constraints on path choice. Stimuli were created so that the shortest possible path of apparent motion would be physically impossible. We hoped to identify those characteristics or features of the human body that lead to the perception of biomechanically consistent paths of apparent motion.

\section{Experiment 1: Bodies About Objects}

Is the visual perception of biomechanically consistent apparent motion specific to the way human bodies move relative to themselves, or does it generalize to the way bodies move with respect to inanimate objects? To address this question, we examined whether the tendency to see the shortest path of apparent motion at short SOAs, and the biomechanically consistent path at long SOAs could be generalized to human movement about inanimate objects. We hypothesized that if sensitivity to biological forms and movements extended to the perception of human movement relative to the outside world, then observers might perceive 
paths of apparent motion that are consistent with normal human movements about inanimate objects. On the other hand, if our sensitivity to biological motion reflects the activity of an actor-centered system, then observers might only perceive paths of apparent motion consistent with the ways in which humans move relative to themselves; that is, as if they were always in isolation. We tested this hypothesis by comparing the perceived paths of apparent motion between two paired classes of stimuli.

The first class of stimuli, termed body about body, consisted of pairs of pictures of a human model moving one of her limbs about either side of some part of her body. We sequentially presented these picture pairs to observers in a manner that essentially replicated an earlier study by Shiffrar and Freyd (1990). We called this first condition body about body because the interpretation of apparent motion required the perception of a human limb to move either through or around another part of the human body. The "through" path of motion was the shortest possible path and was physically impossible. The "around" path of motion was physically possible, but it covered a relatively longer distance. Thus, these around paths violated the shortest path constraint. The purpose of this condition was to determine a baseline for comparison with the results of our second condition. The second condition, termed body about object, again consisted of pairs of images of a human model. However, in this condition we replaced the part of the body about which the limb moved in the previous condition with an inanimate object having approximately the same size and orientation.

The manner in which we interact with objects in the physical world is crucial for our survival and may influence our visual perception (Shepard, 1984). Therefore, we predicted that observers should perceive paths of apparent motion that are consistent with the ways in which humans move with respect to nonbiological objects. That is, we predicted that as the SOA increases, the probability of perceiving the physically possible around path in the body about object condition should also increase. For the body about body condition, we also hypothesized that, as in previous studies, observers should be increasingly likely to perceive biomechanically consistent paths of apparent motion with increasing SOA. We manipulated SOA because it is thought to be most directly related to the apparent motion path curvature (Farrell, 1983; Proffitt et al., 1988). Whereas some previous research regarding the apparent motion of points of light has not produced evidence supportive of a solidity constraint (Berbaum \& Lenel, 1983), other research using more complex stimuli has suggested that observers can perceive paths of apparent motion consistent with the solidity of the human body (Shiffrar \& Freyd, 1990). By comparing the perceived paths of apparent motion across the body about body and the body about object conditions, we could test whether the perception of biomechanically consistent paths of apparent motion was specific to human movement in isolation or generalized to human movement and interaction with the physical world.

\section{Method}

Observers. Sixteen naive Rutgers University undergraduates participated in this experiment for credit toward a class requirement. Observers were tested individually.

Stimuli. All stimuli were presented on a Macintosh 21 in. $(53.34 \mathrm{~cm})$ color display having a $1,152 \times 870$ pixel resolution and a $40 \times 30-\mathrm{cm}$ screen size. A Macintosh Quadra 950 equipped with a VideoSpigot NuBus Digital-Video Frame Grabber was used to create the stimuli, control their presentation, and record observers' responses.

Pairs of full-color images of a human body were created by videotaping a model in different poses. The output of the video camera was captured by the VideoSpigot card on the Macintosh and converted to digital picture files. Screenplay and Quicktime software for the Macintosh were then used to select, edit, and save pairs of pictures. When presented on the monitor, each captured image was $8 \mathrm{~cm}$ high $\times 10 \mathrm{~cm}$ wide and subtended a visual angle of $5.68^{\circ} \times 7.08^{\circ}$ from the observer's viewing distance of $80.5 \mathrm{~cm}$. Each picture pair consisted of the human model in a fixed position except for one limb that was positioned on either side of the model's body or an inanimate object. A competitive strategy was used by creating the stimuli such that the shortest path of motion was physically impossible because it would require a human limb to pass through an occluding solid. A physically possible movement required the observers to perceive human limbs moving around an occluding object or body. These curved around paths were always longer than the shortest possible straight through path.

Seven image pairs were used in the body about body condition. In each picture pair, the human model remained stationary except for the movement of a limb about either side of some part of the model's body. Three of the pairs involved movement of a leg and four of the pairs included movement of an arm or hand. These stimuli included motion of the right foot about the left foot, the left foot about the right ankle, the right foot about the left knee, the right hand about the face, the right hand about the left knee, the right hand about the waist, and the right arm about a bent right knee, while the model was seated (front view). An example of a body about body picture-pair stimulus is shown in Figure 1.

The body about object condition again consisted of seven image pairs individually matched to those in the body about body condition. The only difference between the two sets of pictures was that in the body about object condition, the motion of the limbs was about an inanimate, nonbiological object instead of part of the model's body. For each picture pair, the inanimate object had approximately the same two-dimensional orientation as the body part that it replaced in its corresponding body about body stimulus. Also, for each picture pair in the body about object condition, care was taken to ensure that the body part about which the limb had moved in the body about body condition was clearly visible. Therefore, the inanimate object was placed such that it was adjacent to and did not occlude the body part it replaced. In all the picture pairs, the model remained stationary except for the change of a limb position. The change in limb orientation across the two frames of a stimulus was approximately the same for each body about object stimulus and its corresponding body about body stimulus. The body about object stimuli included movement of the right foot about a box, the left foot about a box (side view), the right foot about a box at the height of the left knee, the right hand about a small box, the right hand about a corner of a chair's backrest, the right hand and wrist about a box positioned at head level, and the right hand about a box while the model was seated. A sample body about object stimulus is shown in Figure 2. 

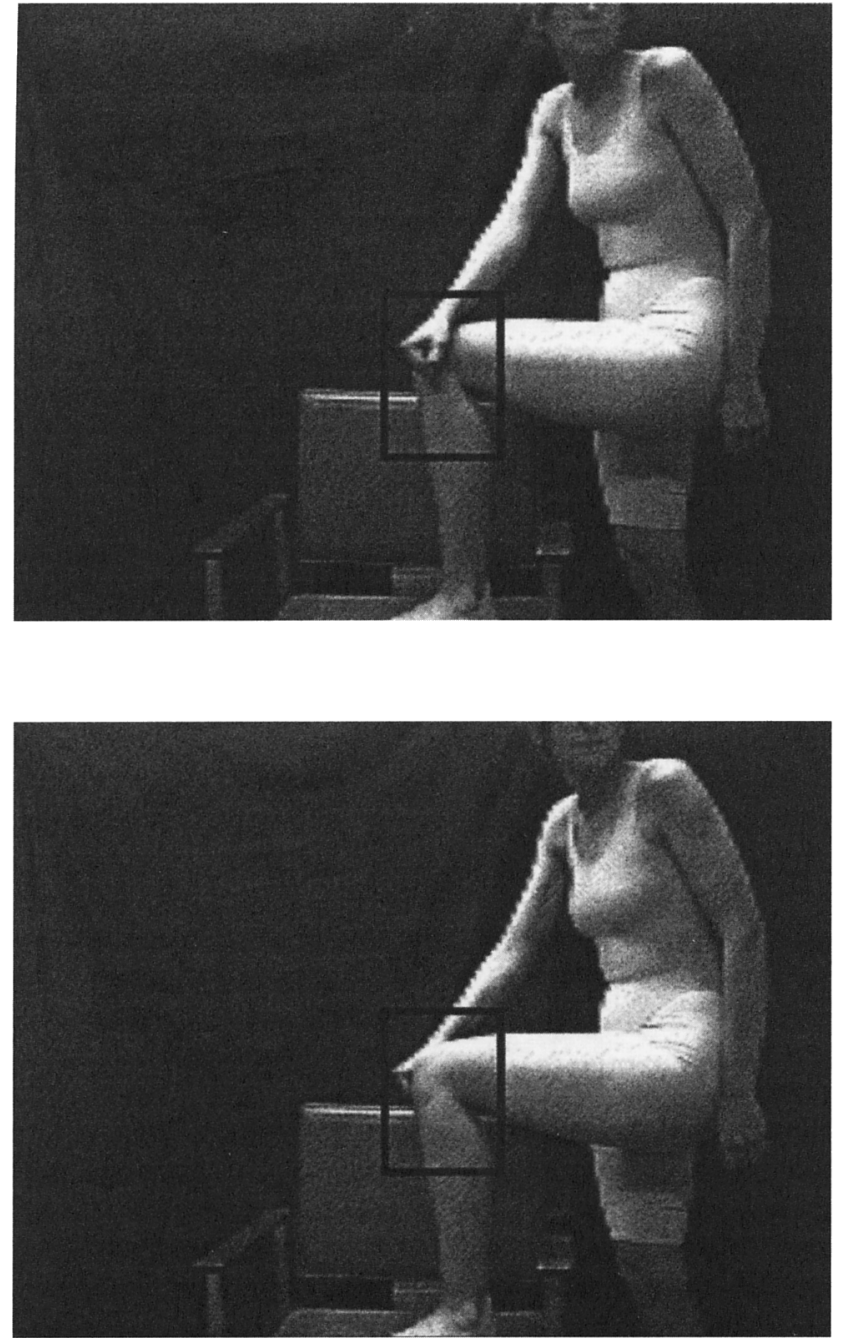

Figure 1. A picture-pair stimulus used in the body about body condition of Experiments 1 and 2 . The model remained stationary except for the displacement of the right hand about the left knee. For Experiment 3, only the area within the black outlined rectangles was visible, and the remaining part of the picture pair was not visible.

The temporal display rate was controlled by Macromedia Director version 3.1 software. The minimum SOA was $133 \mathrm{~ms}$ and the remaining three SOAs were created by adding $133 \mathrm{~ms}$ to each new level. Thus, the SOA levels were 133, 266, 400, and $533 \mathrm{~ms}$. For each SOA level, the length of the stimulus duration (SD) equaled the interstimulus interval (ISI). These temporal values were selected from pilot studies because they provided the most robust apparent motion. It should be noted that these SOAs are much longer than those used in traditional studies of relatively simplistic displays. However, early apparent motion studies demonstrated that the use of complex, meaningful displays extends the spatiotemporal window within which apparent motion is perceived (DeSilva, 1926).

In addition to the 14 image pairs, observers were also presented with four picture pairs displayed at 133 or 533 -ms SOAs as practice trials. The practice trial picture pairs consisted of inanimate objects or human models that were different from those used in the experimental trials. As in the subsequent experimental trials, observers reported whether their perceived paths of apparent motion passed through or around an occluder. The practice trials were used to ensure that every observer understood and felt comfortable with the task before beginning the experimental trials.

Design. In a within-subjects design, each observer was presented with every stimulus at the four different SOA levels. The stimuli were presented in eight blocks with seven stimuli per block. There were four blocks for the body about body condition and four for the body about object condition creating a total of 28 trials per observer in each condition. The presentation of the body about body condition and the body about object condition was counterbalanced with half of the observers viewing the body about body condition first and the other half viewing the body about object condition first. Within each condition, the order of presentation of the picture pairs and the SOAs was randomized. A two-alternative forced-choice procedure was used and the observers indicated whether they perceived the limb to move around or through the inanimate object or body.

Procedure. The observers sat in front of the computer monitor with their chin on a chinrest. The experimenter briefly described the phenomenon of apparent motion to the observers. The observers were then told that they would observe some rapidly flashing
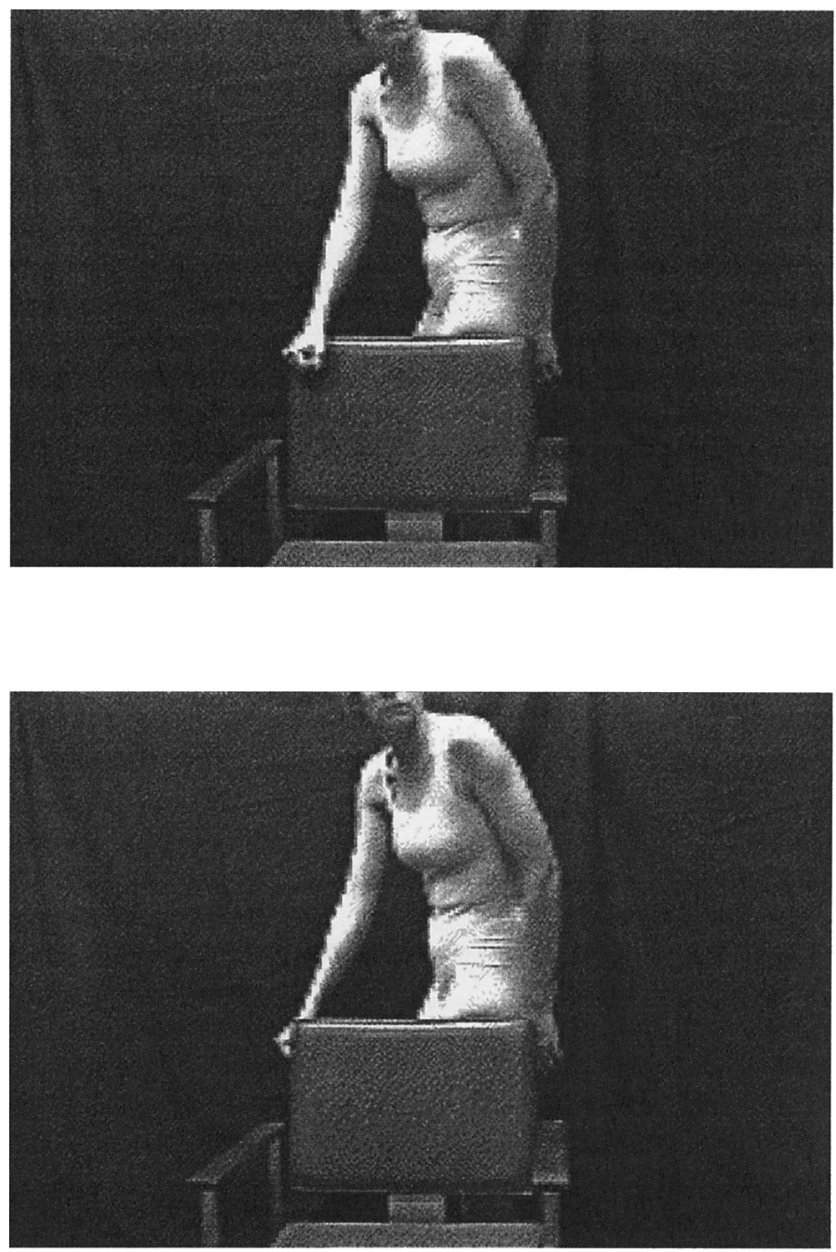

Figure 2. A picture-pair stimulus from the body about object condition. This picture pair is matched to the body about body picture pair shown in Figure 1. 
pictures showing a model in different poses and that sometimes, during this flashing, they might observe some kind of motion. Observers were further instructed that they could observe the flashing stimuli for as long as it took them to determine the path of motion. Once they had determined the path of motion, observers used a mouse device to click a stop button located on the monitor just below the flashing pictures. Following this, a blank screen with a "Please Respond" button appeared. At this time, the observers indicated whether they perceived the path of motion to be around or through the inanimate object or the model's body. The observers initiated the next trial by clicking a button with the mouse device. After the end of each block, the experimenter started a new block. Before starting the experiment, every observer completed four practice trials to ensure that they understood the task.

\section{Results}

For each observer, we tallied the number of times a particular path was chosen at each SOA level for each condition. The data displayed in Figure 3 are plotted as the mean percentage of trials during which observers reported seeing the around path of apparent motion at the four different SOA levels. When creating our stimuli, we made sure that the longer possible path was consistent with the limb movement constraints of the human body. Therefore, when observers reported the perception of around paths, they always reported paths consistent with the range of normal human movements rather than some physically impossible path.

For the body about body condition, observers were significantly more likely to report perceiving the biomechani-

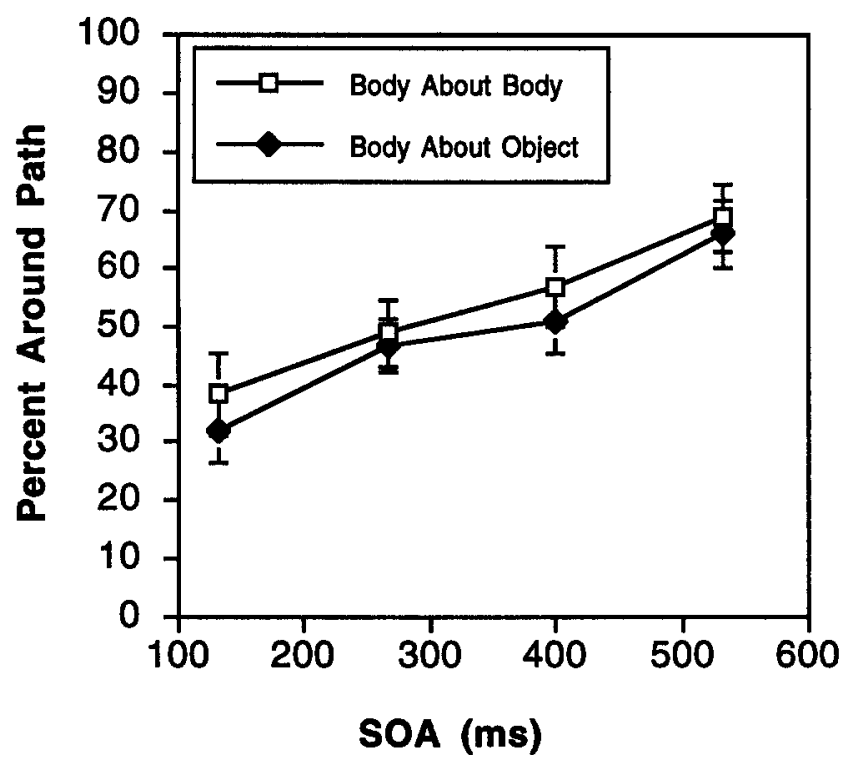

Figure 3. The results of Experiment 1. The data from both conditions are plotted against the four stimulus onset asynchrony (SOA) levels. The error bars represent standard error. The open squares show the body about body condition, and the filled diamonds show the body about object condition. For both conditions, the probability of perceiving the around path increases with SOA. cally consistent around path of apparent motion at the longest SOA level as compared to the shortest SOA level, $t(15)=3.687 ; p<.05$. An analysis of variance (ANOVA) revealed a main effect of SOA level on the frequency of choosing the biomechanically consistent path, $F(3,60)=$ $3.764 ; p<.05$. A regression analysis, in which the SOA level predicted the frequency of choosing the biomechanically consistent path, produced a significant linear trend, $F(1,62)=12.065 ; p<.05$.

Similarly, in the body about object condition, observers were more likely to report perceiving the biomechanically consistent around path of apparent motion at the longest SOA level as compared to the shortest SOA level, $t(15)=$ 4.759; $p<.05$. Again, an ANOVA revealed a main effect of SOA level for the frequency of choosing the around path, $F(3,60)=4.180 ; p<.05$. Finally, a regression analysis in which SOA level was used to predict the frequency of choosing the around path produced a significant linear trend, $F(1,62)=19.534 ; p<.05$.

There was a slightly increased tendency for observers to perceive paths of motion around a human body (body about body) as compared to paths of motion around a solid object (body about object). However, this difference between the two conditions was not statistically significant, $F(1,120)=$ $2.810 ; p>.05$. The condition by SOA interaction was not significant, $F(3,120)=0.268 ; p>.05$. However, a significant main effect for SOA was found with respect to both conditions, $F(3,120)=7.617 ; p<.05$.

\section{Discussion}

These results suggest that the tendency to see biomechanically consistent paths of apparent motion with increasing temporal duration is not limited to the movement of human limbs about the human body. Instead, our visual sensitivity to biological movement appears to be general and incorporates how human bodies move with respect to inanimate objects. This result is consistent with previous point-light walker research. For example, Johansson (1973) demonstrated that a human figure could be easily identified from a point-light model riding an unseen bicycle. Also, when watching point-light displays of actors lifting boxes, observers can judge the weight of a box with quantitative accuracy (Runeson \& Frykholm, 1981). Observers are also able to recognize point-light versions of instrumental actions, such as hammering, ball bouncing, and stirring a bowl, although with less accuracy than locomotory actions (Dittrich, 1993).

It is important to note that observers often perceived through paths of apparent motion even at the longest SOA. Our stimuli were constructed so that the through and around paths of motion were easily discriminable. This resulted in through paths that were much shorter than the biomechanically consistent around paths. Significant differences in distance may have increased the probability of the perception of short through paths of apparent motion.

Taken together, the current results in conjunction with the structure-from-motion findings cited above, suggest that our 
exquisite sensitivity to moving biological forms is particularly robust as it includes simple human movements about nonbiological objects. Thus, our perception of apparent biological motion does not appear to reflect the activity of a strictly isolated or modular system that analyzes animal movements independent of nonbiological objects.

\section{Experiment 2: Objects About Bodies}

What characteristic of the human form causes the perception of biomechanically consistent paths of apparent motion at long temporal intervals? Is a complex, realistic representation of the human form including all of the texture, elasticity, orientation, position, cues and so on associated with the human body required for the perception of apparent biological motion? Or is a subset of all of the human body features sufficient to trigger the perception of apparent biological motion? If sensitivity to biological motion requires the presentation of highly realistic representations of biological forms, then this sensitivity should be restricted to the perception of moving human or animal limbs. On the other hand, sensitivity to biological motion may extend to the interpretation of any apparent motion display containing as little as the limb orientations and positions associated with normal human movement (Shiffrar, Lichtey, \& Heptulla Chatterjee, 1995).

To address these questions, we asked whether observers would continue to perceive paths of apparent motion around the human body when an inanimate object, rather than a human limb, was positioned on either side of the human body. Once again, we compared the perceived paths of apparent motion between two paired classes of stimuli. The first stimulus condition was identical to the body about body condition of Experiment 1 and was used to determine baseline levels. That is, the body about body condition consisted of picture pairs of a human model with one limb displaced about either side of some part of the model's body. In the second condition, called object about body, we replaced each apparently moving limb with a wooden board having approximately the same length and orientation as that limb. Each wooden board was positioned on either side of the stationary human model's body. We hypothesized that, as in previous experiments, observers would be increasingly likely to perceive the longer, biomechanically consistent paths of apparent motion with increasing SOAs in the body about body condition. The critical test concerned the perceived paths in the object about body condition. If the perception of biomechanically consistent paths of apparent motion results from the presentation of a wholly biological form, then no SOA-dependent change in path perception should be seen for the motion of inanimate, nonbiological objects. On the other hand, if only position and orientation cues are needed, then observers should continue to perceive around paths of apparent motion with increasing SOA when nonbiological objects are positioned so that they replicate the limb positions and orientations of normal human movements.

\section{Method}

Observers. Sixteen naive Rutgers University undergraduates participated in this experiment for credit toward a class requirement. Observers were tested individually. None of the observers from Experiment 1 participated in this experiment.

Stimuli and procedure. The apparatus was the same as that used in Experiment 1. The stimuli in the body about body condition were identical to those in the body about body condition of Experiment 1 . The object about body condition consisted of seven picture pairs individually matched to each of the seven body about body picture pairs. The only difference between the two sets of pictures was that in the object about body condition, an inanimate object, rather than a human limb, moved about some part of the model's body. The inanimate object was a wooden board cut to match the length of the limb that it replaced. For each picture pair, the wooden board had approximately the same position and orientation as the moving limb it replaced. The matching procedure consisted of videotaping the human model while the output images were presented on the computer screen. The computer screen simultaneously displayed one of the body about body picture pairs. For each object about body picture pair, the model was positioned so that each pose maximally overlapped, as measured by the eye, with the pose from a body about body picture pair. Then, a wooden board was placed in approximately the same position as the displaced limb and appeared to float in space without support. This ensured that motion percepts were not influenced by the presence of an apparent joint or point of intersection in the picture. The model was then videotaped with the board.

In summary, every picture pair illustrated a human model who remained stationary except for the change of position of a limb or wooden board, depending on the condition. Again, the wooden board was positioned so that it had approximately the same orientation, length, and position as the replaced limb. The object about body stimuli included the displacement of a wooden board about the left foot, the left ankle, the left knee, the face, the left knee (side view), the waist, and the right knee, while the model was seated. An example of the object about body stimuli is shown in Figure 4.

Stimuli were shown at each of the four different SOA levels: $133,266,400$, and $533 \mathrm{~ms}$. The length of the SD always equaled the length of the ISI so that each equaled one half of the SOA. The experimental design and procedure were identical to that of Experiment 1 . The same competitive strategy was used. That is, the stimuli were created so that the shortest path of motion required the perception of a physically impossible movement; that is, either a human limb or a wooden board passing through a human body. According to a within-subjects design, all the observers viewed all of the stimuli at each of the four randomly assigned SOAs. The stimuli for each condition were presented in four blocks with seven trials in each block. Each observer reported whether they perceived the displaced board or limb to move around or through the human model's body. In addition to the 14 picture pairs, observers were also presented with four picture pairs as practice trials. These practice trials were identical to the practice trials of Experiment 1. At the conclusion of the practice trials, all observers reported that they understood and felt comfortable with the task before the experimenter initiated the experimental trials.

\section{Results}

The results, shown in Figure 5, suggest that orientation and position cues may play an important role in the perception of biological apparent motion. The data are again 

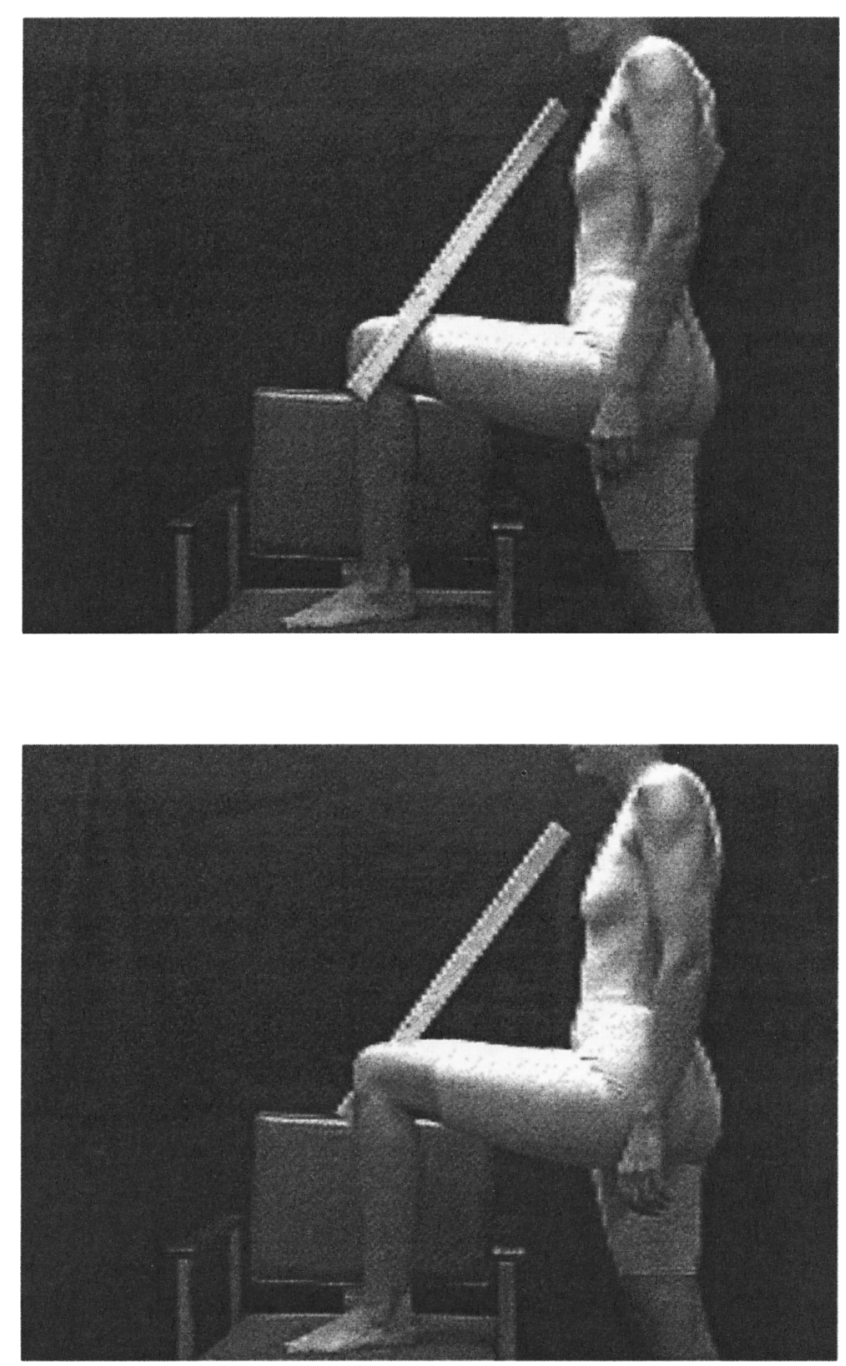

Figure 4. An example of a picture-pair stimulus from the object about body condition of Experiment 2 . This picture pair is matched to the picture pair shown in Figure 1. In each picture the model remained stationary and a wooden board changed position about either side of the model.

plotted as the mean percentage of trials during which observers perceived paths of apparent motion around the human model at the four SOA levels.

For the body about body condition, observers were significantly more likely to report perceiving the biomechanically consistent around path at the longest SOA level as compared with the shortest SOA level, $t(15)=5.258, p<$ .05 . An ANOVA revealed a main effect of SOA level on the frequency of choosing the around path, $F(3,60)=6.240$, $p<.05$. A regression analysis, in which SOA level predicted frequency of choosing the around path, produced a significant linear trend, $F(1,62)=17.559, p<.05$.

Similarly, in the object about body condition, observers were more likely to report perceiving the physically consistent around path of apparent motion at the longest SOA level as compared with the shortest SOA level, $t(15)=$
$2.947, p<.05$. Again, an ANOVA revealed a main effect of SOA level on the frequency of choosing the around path, $F(3,60)=3.082, p<.05$. Finally, a regression analysis in which SOA level was used to predict the frequency of choosing the around path produced a significant linear trend, $F(1,62)=13.605, p<.05$.

There was a slightly increased tendency for observers to perceive a human limb, as compared with a nonbiological object, move around the human body. However, this difference between the body about body and object about body conditions was not statistically significant, $F(1,120)=$ $1.519, p>.05$. Similarly, the Condition $\times$ SOA interaction was not significant, $F(3,120)=0.488, p>.05$. However, a significant main effect for SOA was found with respect to both conditions, $F(3,120)=8.696, p<.05$.

\section{Discussion}

The results suggest that the perception of the longer around paths of motion at longer SOAs may not require the presentation of complex, highly realistic representations of the human body. When the moving human limb was replaced with a nonbiological wooden board having the same length, orientation, and position, perceived paths of motion did not change significantly. For both the object about body and the body about body conditions, the probability of perceiving the around path of motion increased as the temporal interval increased. Because the wooden boards and human limbs shared the same orientations, positions, and solidity, these results suggest that some or all of these

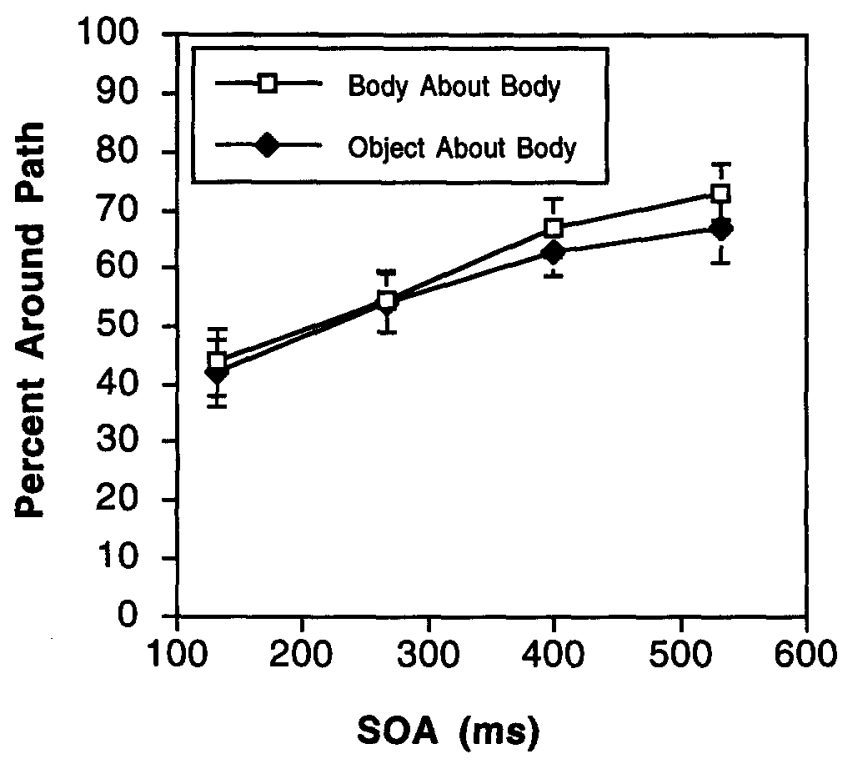

Figure 5. The results from Experiment 2. The data for each condition are plotted against the four stimulus onset asynchrony (SOA) levels. The error bars represent standard error. The open squares show the body about body condition, and the filled diamonds show the object about body condition. For both conditions, the probability of perceiving the around path increases with SOA. 
factors may be sufficient for our perception of biological motion.

In Experiments 1 and 2, we found a main effect for SOA in the body about body conditions as well as the body about object and the object about body conditions. Therefore, our results suggest that visual sensitivity to biological motion can extend to the motion of biological forms about nonbiological objects as well as the motion of nonbiological objects about biological forms. We then examined the characteristics specific to the biological form that lead to this main effect for SOA. To this end, we conducted a new series of experiments to determine whether the global form of the human body, local limb orientation, local limb position, surface curvature, and/or solidity were responsible for our SOA effect.

There are at least three possible explanations for the perception of around paths at long SOAs. The first possibility involves the body about body and object about body conditions in which a limb or stick moved about a human body. A characteristic of the human form is its surface curvature. Curved surfaces can lead to the perception of curved paths of apparent motion at long SOAs (Shepard \& Zare, 1983). Thus, one potential explanation for our results is that the use of a smoothly curved body as an occluder led to the increased perception of curved around paths with increasing SOAs.

Another potential explanation is that the tendency to perceive longer paths with increasing SOAs may result from orientation and solidity cues alone. Because solidity is a property shared by many objects, the results of Experiments 1 and 2 might simply reflect the application of a general solidity constraint. Moreover, several studies have suggested that orientation cues can lead to the perception of curved paths of apparent motion (Foster, 1975; Hecht \& Proffitt, 1991; McBeath \& Shepard, 1989; Proffitt et al., 1988).

A third possible explanation depends on the fact that all of the stimuli in Experiments 1 and 2 included a human form. The global hierarchy of limb orientations and position cues specific to the human form may have led to the perception of longer paths of motion at the longer temporal durations. For purposes of clarity, we define local orientation as the orientation of a single limb or wooden board and global orientation as the ensemble of the orientations of all of the limbs and trunk of the human body.

\section{Experiment 3: Partial Body}

Is the surface curvature of an occluding human body sufficient for the perception of longer, biomechanically consistent paths of apparent motion with increasing SOAs? We addressed this question by presenting subsections of the body about body stimuli used in Experiments 1 and 2 . These reduced stimuli, called partial body stimuli, only depicted the displaced and occluding body parts. This manipulation maintained the curvature of the occluding surface as well as the position, orientation, and apparent solidity of the occluding and displaced limbs. However, these subsections eliminated the global hierarchy of limb orientations and positions of the human body. The purpose of this experiment was to determine whether a main effect of SOA would prevail in the absence of a global hierarchy of orientation and position cues representing a human body.

\section{Method}

Observers. Sixteen naive Rutgers University undergraduates participated in this experiment for credit toward a class requirement. Observers were tested individually. None of the observers had participated in Experiments 1 or 2.

Stimuli and procedure. The apparatus was the same as that used in the previous experiments. Once again, the stimuli consisted of picture pairs displayed in an apparent motion paradigm. Adobe Photoshop software version 2.0.1 was used to create seven pairs of partial body stimuli from the body about body stimuli. The body about body images were altered so that only the moving limb and occluding body part were visible. The remainder of each image was filled with black pixels so that all other body parts were invisible. The visible region of each stimulus subtended $1.1^{\circ} \times$ $1.6^{\circ}$ of visual angle. The rectangular outline superimposed on Figure 1 illustrates a partial body stimulus. As before, each picture pair illustrated the movement of a human limb on either side of a body part. Each observer viewed all seven partial body stimuli at four different SOA levels. The stimuli were presented in two blocks having 14 trials each. The order of picture-pair presentation and SOAs were randomized. As before, the four possible SOAs were 133, 266, 400, and $533 \mathrm{~ms}$. The same procedure from Experiment 1 was used. Again, observers indicated whether they perceived the displaced limb to move around or through the intervening stationary body.

\section{Results and Discussion}

The results of this study, shown in Figure 6, suggest that curvature of the occluding surface cannot account for the SOA effect. The data are again plotted as the mean percentage of trials during which observers reported perceiving around paths of apparent motion. Observers were no more likely to perceive the around path at the longest SOA level as compared to the shortest SOA level, $t(15)=1.775 ; p>$ .05 . An ANOVA revealed no effect of SOA level on the frequency of choosing the around path of motion, $F(3$, $60)=1.316 ; p>.05$. A regression analysis, in which the SOA level predicted the frequency of choosing the around path, did not produce a significant linear trend, $F(1,62)=$ $2.266 ; p>.05$.

These results suggest that surface curvature is not sufficient for the perception of biomechanically consistent around paths at long SOAs. The stimuli in this experiment had the same local curvature as the body about body stimuli from the previous studies. Yet, there was no significant increase in the perception of around paths with increasing SOA in this experiment. The conclusion that surface curvature cannot explain the perception of apparent biological motion is supported by the body about object condition of Experiment 1. In that condition, a human limb always moved about an inanimate, rectangular object. Even though these rectangular occluders did not have smoothly curved 


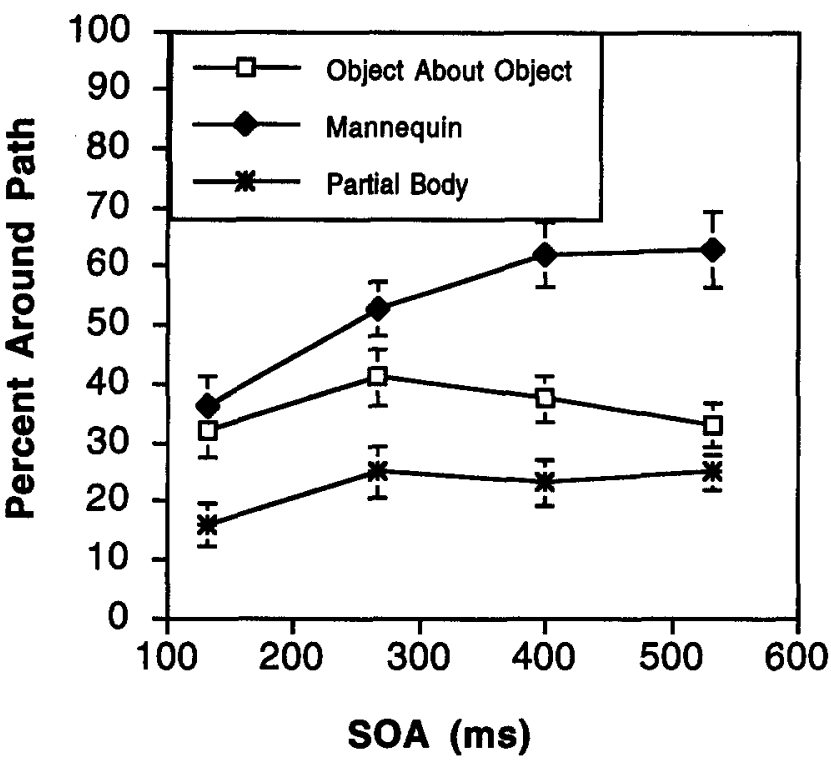

Figure 6. The results from Experiments 3, 4, and 5. The data for each experiment are plotted against the four stimulus onset asynchrony (SOA) levels. The error bars represent standard error. The asterisks show the partial body experiment, the open squares show the object about object experiment, and the filled diamonds show the mannequin experiment results. In the partial body and object about object conditions, the probability of perceiving the around path did not change with SOA. In the mannequin experiment, the probability of perceiving the around path increased with SOA.

surfaces, observers still perceived curved, around paths of apparent motion at long SOAs. The partial body stimuli also controlled for the orientation and position of the displaced and occluding limbs because these were identical to those in the body about body stimuli.

Although the curvature, position, and orientation of the displaced and occluding body parts may not account for the main effect of SOA, we cannot be certain that the partial body stimuli controlled for apparent object solidity. Because our stimuli were highly reduced, the visible surfaces sometimes appeared to lose their solidity. Therefore, we conducted a fourth experiment to determine the extent to which object solidity may have influenced the perception of longer paths with increasing SOAs. Does the tendency to perceive biomechanically consistent, around paths of apparent motion at long SOAs simply result from the presentation of realistic images of solid, three-dimensional (3D) objects having different orientations?

\section{Experiment 4: Objects About Objects}

Is the presentation of realistic images of 3D, differently oriented solid objects sufficient for the increased perception of around paths of apparent motion with increasing SOAs? We addressed this question by presenting observers with images of solid objects arranged so that the objects reproduced the orientation and position of the displaced and occluding limbs of the body about body stimuli. The objects were wooden boards and blocks cut to the same lengths and placed in the same positions and orientations as the displaced limb and stationary occluder limb. These so called object about object stimuli preserved the same local orientation and position cues as well as the solidity of the human body photograph stimuli. However, because only two of the human model's limbs were reproduced, the global form of a human body was absent from these stimuli. Thus, the inanimate objects were always clearly nonhuman, whereas at the same time, their configuration replicated a portion of the human form used in the earlier experiments.

The results of Experiments 1 and 2 demonstrated that observers can perceive biomechanically consistent, around paths of apparent motion at long SOAs in the presence of inanimate objects. By themselves, these results cannot be used to suggest the presentation of a human form leads to the perception of the longer paths of motion. It is entirely possible that the perception of the longer paths only requires the presence of solid, differently oriented objects. Does the presence of the global human form lead to the perception of biomechanically consistent paths of apparent motion?

\section{Method}

Observers. Sixteen naive Rutgers University undergraduates participated in this experiment for credit toward a class requirement. Observers were tested individually. None of the observers had participated in any of the previous experiments.

Stimuli and procedure. The apparatus was the same as that used in Experiments 1-3. The object about object stimuli consisted of seven pairs of pictures displayed in an apparent motion paradigm. The pictures consisted of wooden boards having the same height and width as the displaced and occluder limbs of the human body stimuli used in Experiments 1 and 2. Using Screenplay and Quicktime software for the Macintosh, we were able to match the locations, orientations, and relative sizes of the wooden objects with the displaced and occluder limbs. The remaining human limbs were not represented. All of the wooden blocks and boards presented were clearly nonbiological. As before, each picture pair illustrated the movement of one object about either side of another stationary object. An example of the object about object stimuli is shown in Figure 7.

Each observer viewed all of the object about object stimuli at the four different SOA levels. The stimuli were presented in two blocks having 14 trials each. The order of picture-pair presentation and SOA were randomized. The same procedure and temporal parameters from Experiment 3 were used.

\section{Results and Discussion}

The results of this study, shown in Figure 6, indicate that the presentation of differently oriented, solid objects cannot explain the increased perception of biomechanically consistent apparent motion with increasing SOAs. The data are again plotted as the mean percentage of trials during which observers reported perceiving around paths of apparent motion. In this experiment, observers were no more likely to perceive the around path at the longest SOA level as compared to the shortest SOA level, $t(15)=0.150 ; p>.05$. An ANOVA revealed no effect of SOA on the frequency of 

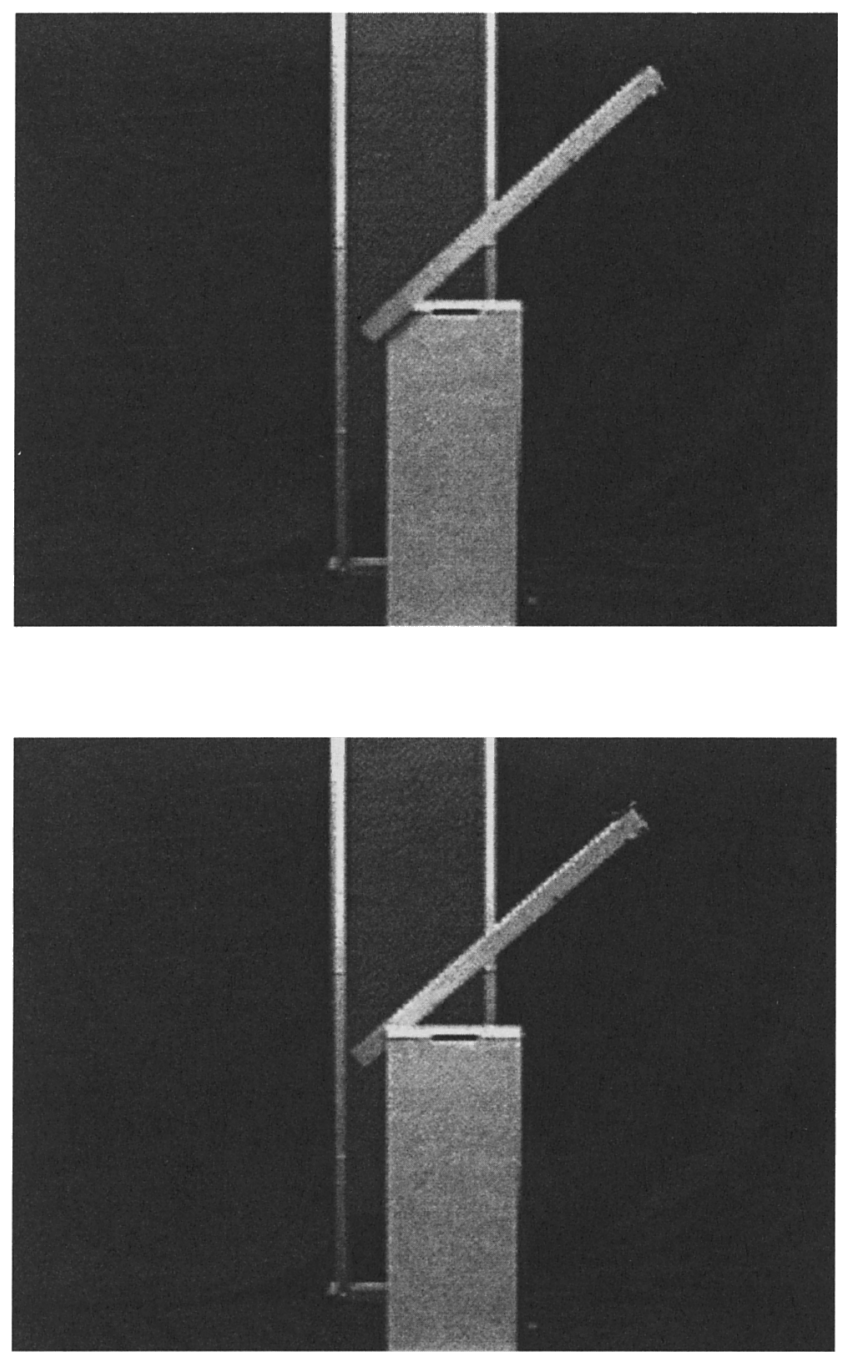

Figure 7. An example of an object about object picture-pair stimulus from Experiment 4 . This picture pair is matched to the picture pair shown in Figure 1. In the two pictures, the wooden board changes position about another board.

choosing the around path of motion, $F(3,60)=1.273 ; p>$ .05 . A regression analysis, in which the SOA level predicted the frequency of choosing the around path did not produce a significant linear trend, $F(1,62)=0.01 ; p>.05$.

These results suggest that whereas the solidity and 3D nature of the human body probably play an important role in biological motion perception, solidity in and of itself, is not sufficient for the increased perception of biomechanically consistent apparent motion with increasing SOAs. The presentation of a solid object that changes orientation and position on either side of an occluder cannot explain our results.

Previous investigators have demonstrated that apparent motion path choice can be influenced by the constraints of object permanence (Gerbino, 1984), rigidity (Berbaum, Lenel, \& Rosenbaum, 1981; Farrell \& Shepard, 1981; Kolers \& Pomerantz, 1971), and occlusion (Anstis \&
Ramachandran, 1985). Part of our results clearly reflect these influences. That is, even at the shortest SOAs, observers perceived the long, around paths for the object about object stimuli on approximately $30 \%$ of the trials. The fact that observers did not perceive the shortest paths of motion during all of these trials may reflect the use of solidity and occlusion constraints in the perception of these stimuli. Most of the literature suggests that these constraints reflect the activity of the long-range apparent motion system that requires relatively long temporal durations (Braddick, 1980; Farrell, 1983). The shortest SOA level used in the current experiments lies well within the temporal range required for long-range apparent motion (Braddick, 1980). Thus, the significant percentage of reported around paths at the shortest SOA in the object about object condition may have resulted from the use of generalized solidity and occlusion constraints because our stimuli were extremely rich in solidity and depth cues.

However, the above constraints, which are not specific to biological organisms, cannot fully explain our results. Specifically, the use of solidity and occlusion constraints cannot explain the increased perception of biomechanically consistent paths of apparent motion with increasing SOAs. This aspect of our results may be most parsimoniously explained by the difference in the global configuration of the stimuli. Therefore, in our final experiment we tried to determine if the global orientation of the human form led to the perception of biomechanically consistent around paths at longer SOAs. We created nonbiological renditions of our human body stimuli to determine if it was the presentation of a hierarchy of limb orientations and positions associated with human movements that led to the increased perception of around paths of apparent motion with increasing SOAs.

\section{Experiment 5: Mannequin}

We created a new set of stimuli depicting the global structure of the human body out of nonhuman material. Stimuli were created by videotaping a wooden mannequin posed in approximately the same positions as the human model in the body about body stimuli. In each picture pair, one of the mannequin's limbs was positioned on either side of some part of its body. Thus, whereas the human body, per se, was absent from these stimuli, the solidity and global and local form of the human body were preserved.

\section{Method}

Observers. Sixteen naive Rutgers University undergraduates participated in this experiment for credit toward a class requirement. Observers were tested individually. None of the observers had participated in any of the previous experiments.

Stimuli and procedure. The apparatus was the same as that used in the previous experiments. The stimuli consisted of seven picture pairs individually matched to those in the body about body condition of Experiments 1 and 2. The mannequin picture pairs were constructed by videotaping a wooden mannequin (model type F1 "Manikin" by Winsor \& Newton) in different positions. The mannequin was videotaped in a zoomed-in or enlarged scale so 
that its image subtended the same visual angle as the human model in the corresponding body about body picture pair stimulus. In each mannequin picture pair, the mannequin remained stationary except for the displacement of one of its wooden limbs on either side of some part of its body. An example of the mannequin stimuli is shown in Figure 8.

Each observer viewed all of the mannequin stimuli at the four different SOA levels. The stimuli were presented in two blocks having 14 trials each. The order of picture-pair presentation and SOA were randomized. The same spatiotemporal parameters and procedures from Experiment 3 were used. Observers indicated whether they perceived the displaced mannequin limb to move around or through the mannequin body.

\section{Results and Discussion}

In this experiment, observers were more likely to report perceiving the around path of apparent motion at the longest
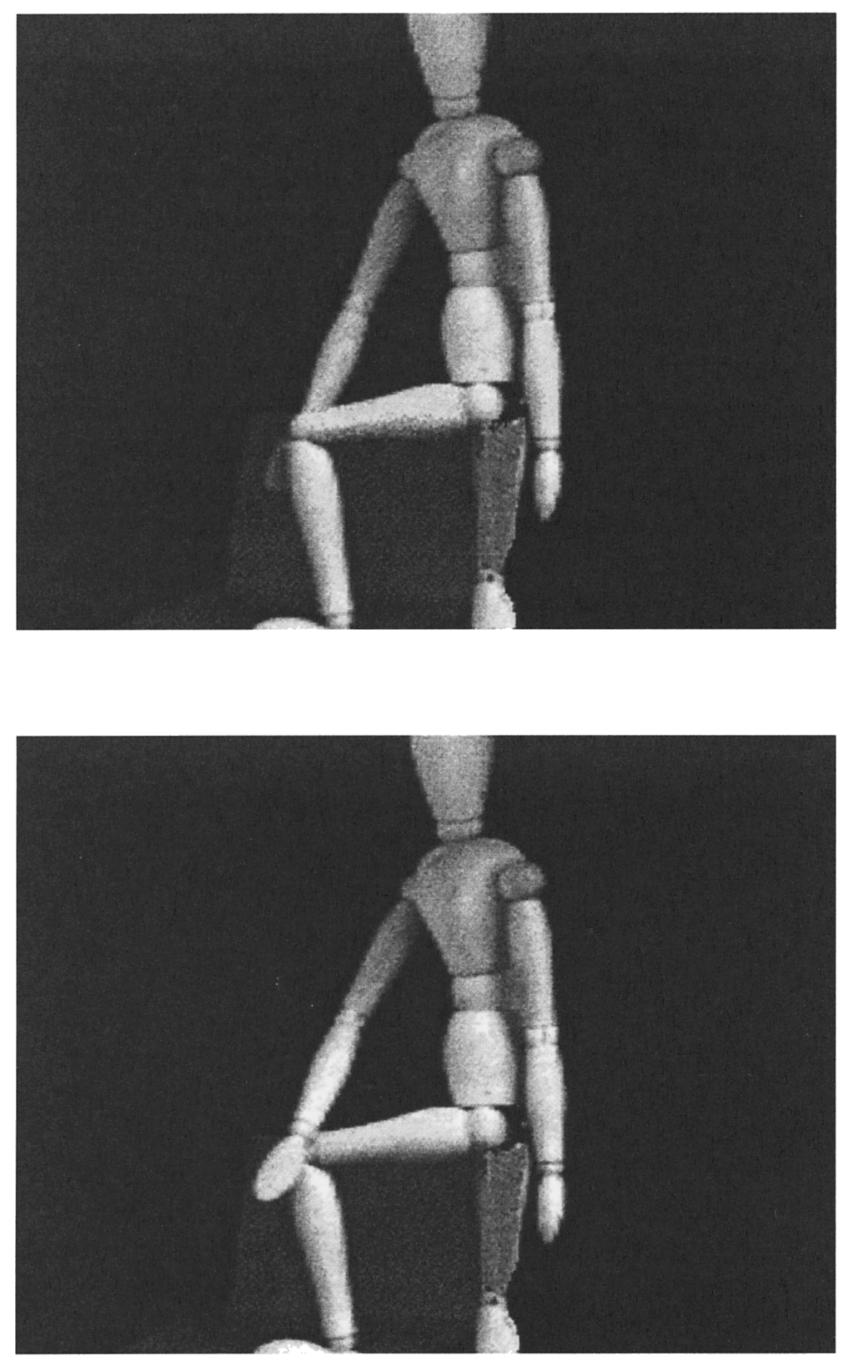

Figure 8. An example of a mannequin picture-pair stimulus from Experiment 5 . This picture pair is matched to the picture pair shown in Figure 1. In the two pictures, the mannequin remains stationary except for the change in position of the right hand about the left knee.
SOA level as compared to the shortest SOA level, $t(15)=$ $3.226, p<.05$. An ANOVA revealed a main effect of SOA level for the frequency of choosing the around path, $F(3$, $60)=9.566, p<.05$. Finally, a regression analysis in which SOA level was used to predict the frequency of choosing the around path produced a significant linear trend, $F(1,62)=$ $25.511, p<.05$.

Like the object about object stimuli, the mannequin stimuli consisted of solid, 3D objects in different orientations. However, the mannequin stimuli also contained a larger human-body context. That is, the displaced wooden appendages were presented as part of a humanlike form rather than in isolation as in the object about object experiment. Thus, the presence of the human form context is associated with the increased perception of biomechanically consistent around paths with increasing SOAs. These results suggest that there may be something special about the global human form that enhances the perception of biomechanically consistent paths of motion.

The results also indicate that sensitivity to biological motion may be evoked by form cues that are not animate or biological in nature. Rather, a global ensemble of limb orientations and position cues of the human form is sufficient for the perception of apparent biological motion. Taken together, the results of Experiments 3, 4, and 5 suggest that global processing occurs in the interpretation of biological forms. This conclusion is consistent with previous research on the perception of point-light walkers (Bertenthal \& Pinto, 1994). Our results are also consistent with Cutting's (1981) findings that observers can recognize computer-generated point-light walkers even though the information in these computer-generated stimuli is not as natural as human gait. Similarly, in the current experiment, in the absence of a realistic representation of the human form, a highly simplified mannequin form was sufficient for the perception of longer paths at the longer SOAs.

\section{General Discussion}

We conducted a series of experiments examining the perceived paths of apparent motion for human figures and nonbiological objects. Previous research suggested that under certain temporal conditions, observers perceive paths of apparent motion that are consistent with the movement limitations of the human body (Shiffrar \& Freyd, 1990; 1993). In the present set of experiments, we were interested in whether the perception of biomechanically consistent apparent motion extended to human movements about nonbiological objects. We also investigated the extent to which factors such as orientation, solidity, and curvature were responsible for the perception of these biomechanically consistent paths at the slow display rates.

In Experiment 1, we found that the likelihood of perceiving biomechanically consistent, around paths of apparent motion increased with SOA for both the movement of human limbs about the human body as well as the movement of human limbs about inanimate objects. From these results we concluded that visual sensitivity to moving bio- 
logical forms may extend to the perception of human actors manipulating inanimate objects in the environment. This conclusion is consistent with several structure-from-motion studies demonstrating that observers can accurately identify point-light defined actors interacting with inanimate objects (Johansson, 1973; Runeson \& Frykholm, 1981).

Experiment 2 investigated whether the presentation of wholly biological forms was required for the perception of apparent biological motion. Observers saw pictures of a human model in which one limb was replaced with a wooden stick. With increasing SOA, observers became increasingly likely to perceive the wooden stick move around the model's body. This suggests that the perception of biomechanically consistent paths of apparent motion can result from the simplified presentation of a subset of the numerous characteristics of the human body.

We then conducted a series of experiments to identify which characteristics of the human form are sufficient for the perception of biological apparent motion. In our Experiment 3, observers viewed reduced renditions of the body about body stimuli, such that only the curved intervening body part and the moving limb were visible. In this experiment, observers did not show an SOA effect on the perceived paths suggesting that curvature of the intervening body parts was not responsible for the longer perceived paths at longer temporal intervals. In our Experiment 4, observers viewed a single, solid, nonbiological object displaced about either side of another solid, inanimate object. The solid objects were arranged so that they maintained the local orientation and position cues of a portion of the human body without resembling a global human form. Here again, we found no SOA effect, suggesting that solidity, orientation, and position cues are insufficient for the perception of apparent biological motion. In our final experiment, we created simplified, nonbiological renditions of our body about body stimuli by replicating human stimuli with a wooden mannequin. The perception of around paths increased with SOA in this human body context, suggesting that a global hierarchy of position and orientation cues consistent with the human form may be sufficient for the perception of apparent biological motion. The global analysis of biological motion and forms has also been suggested from structure-from-motion studies (Bertenthal \& Pinto, 1994).

It is important to note that our stimuli were constructed so that the perception of biomechanically consistent paths always resulted in the perception of a curved motion trajectory. Numerous researchers have demonstrated that when an object changes orientation in a two-frame apparent motion display, the perceived motion path is curved and tends to approximate a circle or sphere (Farrell, 1983; Foster, 1975; Hecht \& Proffitt, 1991; Proffitt et al., 1988). Most of the curved paths reported in our experiments were of a very different type than those arising from orientation differences alone. That is, when our observers perceived around paths of motion, the curved paths were concave relative to the moving object and, therefore, were mirror images of the convex paths perceived with differently oriented, nonbiological stimuli.
Another consideration in the perception of longer paths at longer SOAs relates to Korte's third law (Korte, 1915). That is, the length of the optimal perceived apparent motion path increases linearly with SOA. However, we ruled out this possibility previously because observers viewing two-frame apparent motion displays of a human model are more likely to see the most correct, rather than simply longer, paths of apparent motion with increasing SOA (Shiffrar \& Freyd, 1993). Also, in Experiments 3 and 4, the probability of perceiving the longer, around paths of motion did not change with SOAs. The present SOA effect may reflect a need for additional time to access increasingly specific constraints. With minimal processing time, apparent motion interpretations are first constrained by low-level assumptions of object permanence. With increasing time, additional information regarding occlusion, rigidity, and eventually, biomechanical limitations may be invoked to constrain further image interpretation (Shepard, 1981).

Another important issue is the long-standing controversy surrounding the influence of past experience in the perception of stroboscopic motion. Transactional psychologists studied the influence of past experience in the perception of apparent motion and proposed that meaning connotations in a stimulus may influence path perception in stroboscopic displays (Jones \& Bruner, 1954; Toch \& Ittelson, 1956). Similarly, DeSilva (1926) investigated the role of subjective determinants in movement perception and demonstrated that an increase in stimulus complexity favored good stroboscopic motion. Furthermore, DeSilva found that a meaningful, animate pattern provided a clearer perception of movement direction than a meaningless inanimate pattern. Our stimuli were extremely rich in information and were presented in a highly meaningful context. Thus, the observers' knowledge about human movements may have influenced the perception of biomechanically consistent paths of motion with increasing SOAs. Although our results indicate that biomechanical limitations influence the interpretation of apparent motion, they did not address the question of whether this "knowledge" is a result of perceptual learning and past experience or some implicit knowledge of motor system function (Viviani \& Stucchi, 1992). We should also point out an essential difference between our definition of knowledge and what is traditionally referred to as expectancy. Our results cannot be explained in terms of an observer response bias resulting from expectancy because the probability of seeing biomechanically consistent paths of motion increased as SOA increased. If expectancy played a determining role in the perception of the biomechanically consistent paths, then observers should never have perceived the biomechanically impossible through paths of apparent motion at short SOAs.

In recent years, the relationship between the processing of form and motion has received considerable attention. Form and motion were thought to be processed independently by the ventral and dorsal systems, respectively (DeYoe \& Van Essen, 1988; Livingstone \& Hubel, 1988). However, the separation between these two systems may not be complete because both project to the superior temporal sulcus (STS), suggesting that this area may be the site of cooperation 
between "what" and "where" systems (Baizer, Ungerleider, \& Desimone, 1991; Goodale \& Milner, 1992). Cells in the STS of the macaque monkey exhibit an increased rate of firing when presented with displays consisting of precise combinations of biological forms and motions (Perrett, Harries, Mistlin, \& Chitty, 1990). Our behavioral studies are consistent with this cooperative analysis of form and motion, and similarly, they suggest that form as well as motion may play an important role in the interpretation of biological motion.

In conclusion, we propose that a global hierarchy of orientation cues resembling the human form may be required for the perception of biomechanically consistent paths of apparent motion. Johansson $(1973 ; 1975)$ originally proposed that the analysis of point-light walker displays depended strongly on the presence of a hierarchy of pendular motions. Similarly, our results suggest there may be a hierarchy of orientation and position cues present in biological forms that leads to the perception of biomechanically consistent movements.

\section{References}

Anstis, S., \& Ramachandran, V. (1985). Kinetic occlusion by apparent motion. Perception, 14, 145-149.

Baizer, J., Ungerleider, L., \& Desimone, R. (1991). Organization of visual inputs to the inferior temporal and posterior parietal cortex in macaques. Journal of Neuroscience, 11, 168-190.

Barclay, C., Cutting, J., \& Kozlowski, L. (1978). Temporal and spatial factors in gait perception that influence gender recognition. Perception \& Psychophysics, 23, 145-152.

Berbaum, K., \& Lenel, J. C. (1983). Objects in the path of apparent motion. American Journal of Psychology, 96, 491-501.

Berbaum, K., Lenel, J. C., \& Rosenbaum, M. (1981). Dimensions of figural identity and apparent motion. Journal of Experimental Psychology: Human Perception and Performance, 7, 13121317.

Bertenthal, B. I., \& Pinto, J. (1993). Complementary processes in the perception and production of human movements. In $\mathbf{E}$. Thelen \& L. Smith (Eds.), Dynamical approaches to development: Vol. 2. Approaches (pp. 209-239). Cambridge, MA: Bradford Books.

Bertenthal, B. I., \& Pinto, J. (1994). Global processing of biological motions. Psychological Science, 5, 221-225.

Bertenthal, B. I., Proffitt, D. R., Spetner, N.B., \& Thomas, A. (1985). Development of the perception of biomechanical motions. Child Development, 56, 531-543.

Blake, R. (1993). Cats perceive biological motion. Psychological Science, 4, 54-57.

Braddick, O. J. (1980). Low-level and high-level process in apparent motion. Philosophical Transactions of the Royal Society of London, 290, 131-151.

Burt, P., \& Sperling, G. (1981). Time, distance and feature tradeoffs in visual apparent motion. Psychological Review, 7, 171195.

Cutting, J. E. (1981). Coding theory adapted to gait perception. Journal of Experimental Psychology: Human Perception and Performance, 7, 71-81.

Cutting, J. E. (1987). Perception and information. Annual Review of Psychology, 38, 61-90.

Cutting, J. E., Moore, C., \& Morrison, R. (1988). Masking the motions of human gait. Perception \& Psychophysics, 44, 339347.

Cutting, J. E., \& Proffitt, D. (1981). Gait perception as an example of how we may perceive events. In R. Walk \& H. Pick (Eds.), Intersensory perception and sensory integration (pp. 249-273). New York: Plenum.

DeSilva, H. R. (1926). An experimental investigation of the determinants of apparent visual motion. Journal of Experimental Psychology, 37, 469-501.

DeYoe, E., \& Van Essen, D. (1988). Concurrent processing streams in monkey visual cortex. Trends in Neuroscience, 11, 219-226.

Dittrich, W. H. (1993). Action categories and the perception of biological motion. Perception, 22, 15-22.

Farrell, J. E. (1983). Visual transformations underlying apparent movement. Perception \& Psychophysics, 33, 85-92.

Farrell, J., \& Shepard, R. N. (1981). Shape, orientation, and apparent rotational motion. Journal of Experimental Psychology: Human Perception and Performance, 7, 477-486.

Foster, D. H. (1975). Visual apparent motion and some preferred paths in the rotation group SO(3). Biological Cybernetics, 18, 81-89.

Gerbino, W. (1984). Low-level and high-level processes in the perceptual organization of three-dimensional apparent motion. Perception, 13, 417-428.

Goodale, M. A., \& Milner, A. D. (1992). Separate visual pathways for perception and action. Trends in Neuroscience, 15, 20-25.

Green, M. (1986). What determines correspondence strength in apparent motion? Vision Research, 26, 599-607.

He, Z. J., \& Nakayama, K. (1994). Apparent motion determined by surface layout not by disparity or three-dimensional distance. Nature, 367, 173-175.

Hecht, H., \& Proffitt, D. R. (1991). Apparent extended body motions in depth. Journal of Experimental Psychology: Human Perception and Performance, 17, 1090-1103.

Hoffman, D. D., \& Flinchbaugh, B. E. (1982). The interpretation of biological motion. Biological Cybernetics, 42, 195-204.

Johansson, G. (1973). Visual perception of biological motion and a model for its analysis. Perception \& Psychophysics, 14, 201211.

Johansson, G. (1975). Visual motion perception. Scientific American, 232, 76-88.

Johansson, G., von Hofsten, C., \& Jansson, G. (1980). Event perception. Annual Review of Psychology, 31, 27-63.

Jones, E. E., \& Bruner, J. S. (1954). Expectancy in apparent visual motion. British Journal of Psychology, 45, 157-165.

Kolers, P., \& Pomerantz, P. (1971). Figural change in apparent motion. Journal of Experimental Psychology, 87, 99-108.

Korte, A. (1915). Kinematoskopische Untersuchungen. Zeitschrift fuer Psychologie, 72, 194-296.

Kozlowski, L. T., \& Cutting, J. E. (1977). Recognizing the sex of a walker from a dynamic point-light display. Perception \& Psychophysics, 21, 575-580.

Kozlowski, L. T., \& Cutting, J. E. (1978). Recognizing the sex of a walker from point-lights mounted on ankles: Some second thoughts. Perception \& Psychophysics, 23, 459.

Livingstone, M., \& Hubel, D. (1988, May 6). Segregation of form, color, movement, and depth: Anatomy, physiology, and perception. Science, 240, 740-749.

MacArthur, L. Z., \& Baron, M. K. (1983). Toward an ecological theory of social perception. Psychological Review, 90, 215-238.

Mack, A., Klein, L., Hill, J., \& Palumbo, D. (1989). Apparent motion: Evidence of the influence of shape, slant, and size on the 
correspondence process. Perception \& Psychophysics, 46, 201206.

Mather, G., \& West, S. (1993). Recognition of animal locomotion from dynamic point-light displays. Perception, 22, 759-766.

McBeath, M. K., \& Shepard, R. N. (1989). Apparent motion between shapes differing in location and orientation: A window technique for estimating path curvature. Perception \& Psychophysics, 46, 333-337.

Navon, D. (1976). Irrelevance of figural identity for resolving ambiguities in apparent motion. Journal of Experimental Psychology: Human Perception and Performance, 2, 130-138.

Pantle, A. J., \& Petersik, J. T. (1980). Effects of spatial parameters on the perceptual organization of a bistable motion display. Perception \& Psychophysics, 27, 307-312.

Perrett, D., Harries, M., Mistlin, A. J., \& Chitty, A. J. (1990). Three stages in the classification of body movements by visual neurons. In H. B. Barlow, C. Blakemore, \& M. Weston-Smith (Eds.), Images and understanding (pp. 94-107). Cambridge, England: Cambridge University Press.

Proffitt, D. R., Bertenthal, B. I., \& Roberts, J. R. J. (1984). The role of occlusion in reducing multistability in moving point-light displays. Perception \& Psychophysics, 36, 315-323.

Proffitt, D. R., Gilden, D. L., Kaiser, M. K., \& Whelan, S. M. (1988). The effect of configural orientation on perceived trajectory in apparent motion. Perception \& Psychophysics, 43, 465474.

Runeson, S., \& Frykholm, G. (1981). Visual perception of lifted weights. Journal of Experimental Psychology: Human Perception and Performance, 7, 733-740.

Shepard, R. N. (1981). Psychophysical complementarity. In M. Kubovy \& J. Pomerantz (Eds.), Perceptual organization (pp. 279-341). Hillsdale, NJ: Erlbaum.
Shepard, R. N. (1984). Ecological constraints on internal representation: Resonant kinematics of perceiving, imagining, thinking, and dreaming. Psychological Review, 91, 417-447.

Shepard, R. N., \& Zare, S. (1983, May 6). Path guided apparent motion. Science, 220, 632-634.

Shiffrar, M. (1994). When what meets where. Current Directions in Psychological Science, 3, 96-100.

Shiffrar, M., \& Freyd, J. J. (1990). Apparent motion of the human body. Psychological Science, 1, 257-264.

Shiffrar, M., \& Freyd, J. J. (1993). Timing and apparent motion path choice with human body photographs. Psychological Science, 4, 379-384.

Shiffrar, M., Lichtey, L., \& Heptulla Chatterjee, S. (in press). The perception of biological motion across apertures. Perception \& Psychophysics.

Sumi, S. (1984). Upside-down presentation of the Johansson moving light-spot pattern. Perception, 13, 283-286.

Toch, H. H., \& Ittelson, W. H. (1956). The role of past experience in apparent movement: A revelation. British Journal of Psychology, 47, 195-207.

Viviani, P., \& Stucchi, N. (1992). Biological movements look uniform: Evidence of motor-perceptual interactions. Journal of Experimental Psychology: Human Perception and Performance, $18,603-623$.

Webb, J. A., \& Aggarwal, J. K. (1982). Structure from motion of rigid and jointed objects. Artificial Intelligence, 19, 107-130.

Wertheimer, M. (1912). Experimentelle stuidien uber das Sehen von Beuegung. Zeitschrift fuer Psychologie, 61, 161-265.

Received September 21, 1994

Revision received March 22, 1995

Accepted June 5, 1995

\section{Mentoring Program Available for International Scholars}

APA's Committee on International Relations in Psychology is encouraging publication of international scholars' manuscripts in U.S. journals. To accomplish this initiative, the Committee is looking for authors whose native language is not English to work with U.S. mentors. U.S. mentors will help authors bring manuscripts into conformity with English-language and U.S. publication standards.

The Committee also continues to update its mentor list and is looking for U.S. mentors, especially those with translating and APA journal experience. Interested individuals should contact Marian Wood in the APA International Affairs Office, 750 First Street, NE, Washington, DC 20002-4242. Electronic mail may be sent via Internet to mzw.apa@email.apa.org; Telephone: (202) 336-6025; Fax: (202) 336-5919. 